\begin{tabular}{|l|l|}
\hline$G C-4$ & 931 \\
\hline
\end{tabular}

$r$
MILITARY TECHNICAL COLLEGE

CAIRO - EGYPT
1

\author{
THE INFLUENCE OF SAMPLING INTERVAL ON THE \\ PERFORMANCE OF MINIMUM VARIANCE CONTROL SYSTEMS
}

\author{
M. A. KOUTB*
}

\begin{abstract}
The performance of minimum variance control systems using controllers performing with different sampling intervals is considered. The controller considered is the generalized minimum variance controller. The analysis is carried out using the simulation technique. Two simulation models are presented to solve this problem. The presented simulation models are applied on a set of processes identified using different sampling intervals. The results are compared by evaluating some performance index defined for the discrete output and input. The performance of the minimum variance control systems is explained in the extreme cases. These cases include very samll sampling intervals compared with the smallest time constant of the continuous process and very large sampling intervals compared with the smallest time constant of the continuous process. The performance using sampling intervals comparable to the smallest time constant of the continous process is explained as well.
\end{abstract}

\title{
INTRODUCTION
}

The choice of sampling interval is one of the most important design decisions for discrete-time control systems. This is because it is the main factor influencing control performance for any choice of control algorithm Janiszowski [1]. The influence of sampling interval on the performance of control systems using the discrete parameter-optimized controllers, deadbeat controllers and state controllers has been investigated by Isermann [2]. However, Isermann does not discuss the influence of sampling interval on the performance of minimum variance control systems. The minimum variance controller is easy to derive from an identified model of the system. It is also suitable for use in self tuning control Astrom [3]. To determine the influence of sampling interval on minimum variance control by means of simulation, it is

* Doctor Eng., Dept. of Industrial Electronics, Menoufia University, Menouf, Egypt. 
Fecessary to compare the input/output variances for controllers performing with different sampling intervals. It is assumed that a discrete-time linear dynamic shochastic model has been identified from data collected using sampling intervaliseferred to as the basic sampling interval and denoted $\mathrm{T}_{\mathbf{S}}$. The parameters and structure of this model can be calculated for the integer multiples of the basic sampling interval, i.e. $\mathrm{hT}_{\mathbf{S}}$, where $h$ is an integer number. The paper presents two simulation models for the calculation of the input/output variances of the controlled processes for sampling intervals that are integer multiples of the basic sampling interval. In the first simulation model, the input/ output variances for different $\mathrm{hT}_{\mathrm{S}}$ are calculated using the input/ output observations and models for the corresponding $\mathrm{hT}_{\mathrm{S}}$. In the second simulation model, the input/output variances for different $\mathrm{hT}_{\mathrm{s}}$ are calculated using the input/ output observations and models for the basic sampling interval but the controller is designed for different sampling intervals and supplied by output measurements corresponding also to these sampling intervals. The proposed simulation models are applied on a set of processes identified using different basic sampling intervals. The results are then compared on the basis of the discrete output and input variances.

\section{PROCESS MODEL}

Consider a single-input single-output linear dynamic stochastic process whose model has been identified from data collected with sampling interval $\mathrm{T}_{\mathrm{S}}$. This model can be represented by the following difference equation

$$
y(i)=z^{-k} \frac{B\left(z^{-1}\right)}{A\left(z^{-1}\right)} u(i)+\frac{C\left(z^{-1}\right)}{A\left(z^{-1}\right)} e(i)
$$

where, $y(i)$ is the output signal at instant $i, u(i)$ is the input signal at instant $i, z^{-1}$ denotes the backward-shift operator i.e., $z^{-1} y(i)=y(i-1), k$ is the dead time of the identified model and $e(i)$ is white noise of the type $(0, \lambda 2)$. $A\left(z^{-1}\right), B\left(z^{-1}\right)$ and $C\left(z^{-1}\right)$ are polynomials defined by

$$
\begin{aligned}
& A\left(z^{-1}\right)=1+a_{1} z^{-1}+\ldots+a_{n} z^{-n} \\
& B\left(z^{-1}\right)=b_{0}+b_{1} z^{-1}+\ldots+b_{n} z^{-n} b_{0} \neq 0 \\
& C\left(z^{-1}\right)=1+c_{1} z^{-1}+\ldots+c_{n} z^{-n}
\end{aligned}
$$

$C\left(z^{-1}\right)$ is allowed to have roots only on or within the unit circle, Astrom [4]. In the following we will denote the second term in the R.H.S in (1) by d(i), i.e.,

$$
d(i)=\frac{C\left(z^{-1}\right)}{A\left(z^{-1}\right)} \text { e(i) }
$$

The minimum variance control algorithm for the process described by (1) is given by, Astrom [4]

$$
u(i)=\frac{-G\left(z^{-1}\right)}{B\left(z^{-1}\right) F\left(z^{-1}\right)+Q C\left(z^{-1}\right)} y(i)
$$


Fhere $Q$ is a weighting factor, $F\left(z^{-1}\right)$ and $\mathrm{G}_{\left(\mathrm{z}^{-1}\right)}$ are polynomials determined by the identity

$$
C\left(z^{-1}\right)=A\left(z^{-1}\right) F\left(z^{-1}\right)+z^{-K} G\left(z^{-1}\right)
$$

and,

$$
\begin{aligned}
& F\left(z^{-1}\right)=1+f_{1} z^{-1}+\ldots \ldots+f_{K-1} z^{-(K-1)} \\
& G\left(z^{-1}\right)=g_{0}+g_{1} z^{-1}+\ldots+g_{n-1} z^{-(n-1)}
\end{aligned}
$$

where $\mathrm{K}=\mathrm{k}+1$ is the time delay of the control loop including the controller as well, Koutb [5].

\section{SIMULATION MODELS}

In the following we are going to calculate the input/output variances of the controlled process for the basic sampling interval for which the model has been identified, i.e., $T_{\mathbf{S}}$ and to do the same task for the integer multiples of the basic sampling interval, i.e., $\mathrm{hT}_{\mathrm{s}}(\mathrm{h}=1,2, \ldots)$ using simulation technique.

To calculate the input variance for the basic sampling interval $T_{S}$, we have to calculate the parameters of the controller using (5), (4) is then used to calculate u(i). The input variance is calculated using the following equation

$$
\operatorname{var} u(i)=\frac{1}{N} \sum_{i=1}^{N}[u(i)-\bar{u}]^{2}
$$

where $\mathrm{N}$ is the number of samples used and $\overline{\mathrm{u}}$ is the mean of u (i).

The process output at instant $i$ is given by

$$
y(i)=\stackrel{*}{y}(i)+d(i)
$$

where ${ }^{*}(i)$ represents the output of the deterministic part of the identified model and $d(i)$ represents the output of the stochastic part of the identified model at instant $i$. The output variance is calculated using the following equation

$$
\operatorname{var} y(i)=\frac{1}{N} \sum_{i=1}^{N}[y(i)-\bar{y}]^{2}
$$

where $\bar{y}$ is the mean of $y(i)$. Fig. 1 illustrates the case.

As the sampling interval is an integer multiple of $\mathrm{T}_{\mathbf{S}}$, it is possible to formulate two simulation models for the calculation of the input/ output variances of the controlled process.

The First Simulation Model

This model is illustrated in Fig. 2. The parameters of the deterministic part of the identified model and those of the stochastic part are calculated for $h=2,3, \ldots$, using the algorithm described by Niederlinski [6]. The parameters of the controller for a given $h$ are calculated using the parameters of the deterministic and stochastic parts calcLulated for the corresponding $h$. 
$r$

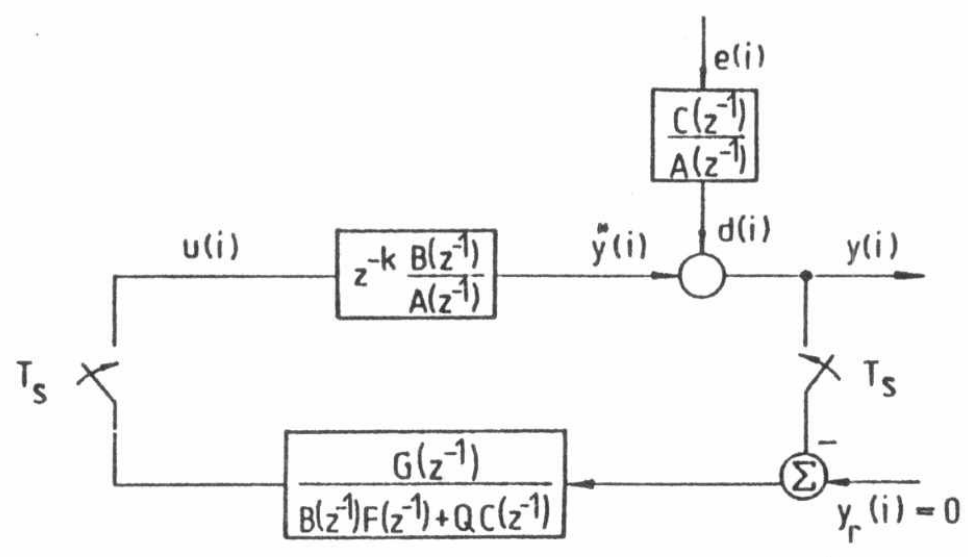

Fig.1. Minimum variance control simulation model using the basic sampling interval $T_{S}$

The input variance is calculated using the following equation

$$
\operatorname{var} u(i)_{h}=\frac{1}{N} \sum_{i=1}^{N}\left[u(i)_{h}-\bar{u}_{h}\right]^{2}
$$

where $u(i) h$ denotes the control signal at instant i, this control signal is calculated every $\mathrm{hT}_{\mathrm{s}}$ interval and $\bar{u}_{\mathrm{h}}$ is the mean of $u(i)_{h}$.

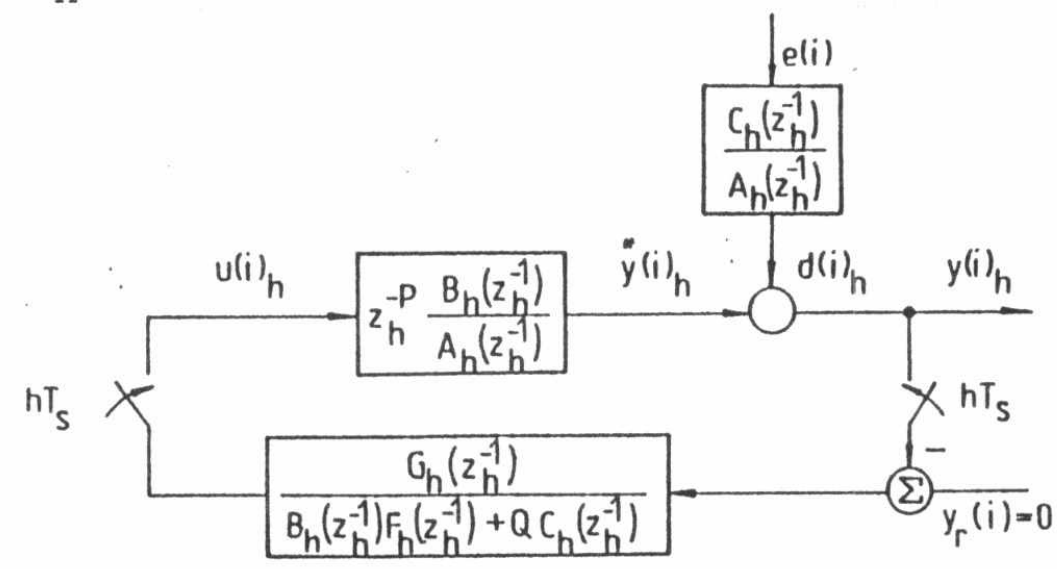

Fig. 2. Minimum variance control simulation model using integer multiples of the basic sampling interval $T_{s}$ for both process and controller

The output variance is calculated using the following equation

$$
\operatorname{var} y(i)_{h}=\frac{1}{N} \sum_{i=1}^{N}\left[y(i)_{h}-\bar{y}_{h}\right]^{2}
$$

where $\mathrm{y}(\mathrm{i})_{\mathrm{h}}=\stackrel{*}{\mathrm{y}}(\mathrm{i})_{\mathrm{h}}+\mathrm{d}(\mathrm{i})_{\mathrm{h}}$ where $\stackrel{*}{\mathrm{y}}(\mathrm{i})_{\mathrm{h}}$ and $\mathrm{d}(\mathrm{i})_{\mathrm{h}}$ denote the output of the deterministic part and the output of the stochastic part respectively at instant $i$, these outputs are calculated every $\mathrm{hT}_{\mathrm{S}}$ interval and $\mathrm{y}_{\mathrm{h}}$ denotes the mean of $\mathrm{y}(\mathrm{i})_{\mathrm{h}}$.

The disadvantage of this model is that we are lossing information by calculating the output variance only at the new sampling instants and not between them although it is possjible to calculate the input output variances always for the 
Fasic sampling interval. The next model explains this idea.

The Second Simulation Model

This model is illustrated in Fig. 5b. The prcoess model corresponds always to the basic sampling interval no matter for what sampling interval we do simulation. The parameters of the controller are calculated exactly as in the previous model, i.e., for each different sampling interval. To interface the process model with the controller, it is necessary to introduce:

A. A discrete sampler, which samples at ${ }^{h} \mathrm{~T}_{\mathbf{S}}$ the output already sampled with $\mathrm{T}_{\mathbf{S}}$.

B. A discrete extrapolator, Fig. 3ar which supplies the process with the same control signals for each $\mathrm{T}_{\mathrm{S}}$ subinterval of the $\mathrm{hT}_{\mathrm{s}}$ sampling interval.

The deterministic and the stochastic parts of the identified model at the basic sampling interval are then used to calculate ${\stackrel{\star}{y_{b}}}_{b}(i)$ and $d(i)$. The output observation at instant $i$ is given by

$$
y_{b}(i)=\stackrel{*}{y}_{b}(i)+d(i)
$$

where $\stackrel{*}{Y}_{\mathrm{b}}(i)$ is the output of the deterministic part and $d(i)$ is the output of the stochastic part at instant $i$. These outputs are calculated every $\mathrm{T}_{\mathbf{S}}$ interval.

The output variance is calculated using the following equation

$$
\operatorname{var} y_{b}(i)=\frac{1}{N} \sum_{i=1}^{N}\left[y_{b}(i)-\bar{y}_{b}\right]^{2}
$$

where $\bar{y}_{b}$ denotes the mean of $y_{b}(i)$.

'rhe input variance is calculated using the following equation

$$
\operatorname{var} u_{b}(i)=\frac{1}{N} \sum_{i=1}^{N}\left[u_{b}(i)-\bar{u}_{b}\right]^{2}
$$

where $u_{b}(i)$ is the output of the extrapolator at instant $i$ and $\vec{u}_{b}^{b}$ is the mean of $u_{b}(i)$.

The main advantage of this model is that we can always caliulate the variances for the basic sampling interval and :herefore are in a position to analyze the influence of sampling interval on the best approximation to the continuous process-the process vith smallest i.e., basic sampling interval.

\section{EXAMPLES}

In the following we present the simulation results for a set of processes identified using different sampling intervals and controlled using minimum variance controllers performing with different sampling intervals. The simulation models already described are used to calculate the input/ output variances for diffecent $\mathrm{hT}_{\mathbf{S}}$. The weighting factor $\mathrm{Q}$ is 
$\Gamma$

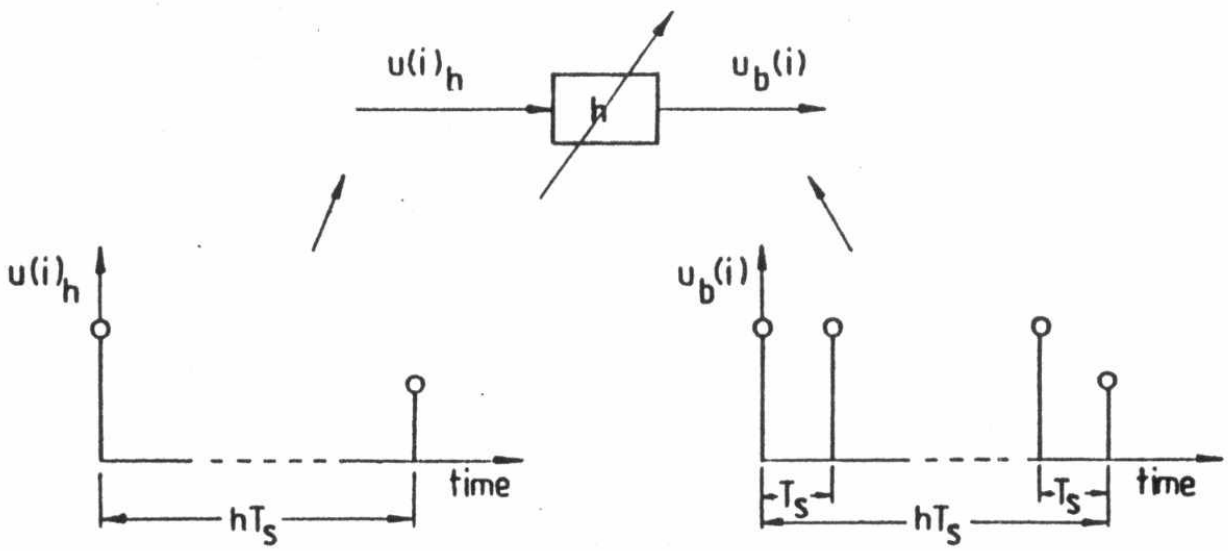

F1g.3a. The discrete extrapolator

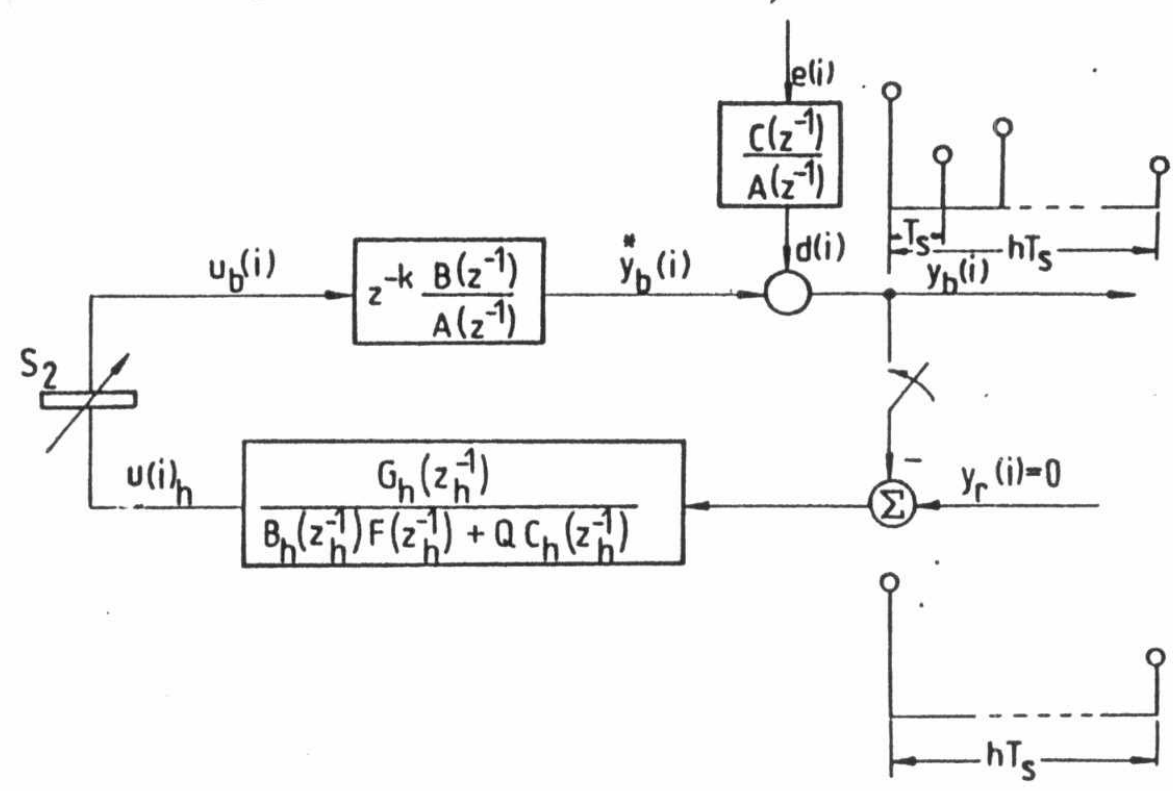

Fig. 3b. Minimum variance control simulation model with the controller parameters calculated at integer multiples of $T_{\mathbf{S}}$ and the process parameters calculated at $T_{\mathbf{S}}$

Example 1

The model

$$
y(i)=z^{-1} \frac{0.01}{1-0.99 z^{-1}} u(i)+\frac{1+0.99 z^{-1}}{1-0.99 z^{-1}} e(i)
$$

represents the model obtained by sampling the continuous process

$$
G(s)=\frac{e^{-\theta s}}{1+T_{1} s}
$$

assuming $\mathrm{T}_{1}=1, \theta=0, \mathrm{~T}_{\mathbf{S}}=0.01$ and assuming that the disturbance is described by

$$
d(i)=\frac{1+0.99 z^{-1}}{1-0.99 z^{-1}} \text { e(i) }
$$


This choice of the disturbance model ensures that a very small 7 sampling interval was used in the identification of this model, Koutb [5]. The parameters of the model (16) as a function of the sampling interval ${ }^{h T_{S}}$ are tabulated in Table 1.

Table 1. Parameters of the model (16) for some $\mathrm{hT}_{\mathbf{S}}$.

\begin{tabular}{|c|c|c|c|c|c|}
\hline $\mathrm{h}$ & $\mathrm{k}$ & $\mathrm{b}_{\mathrm{O}}$ & $\mathrm{a}_{1}$ & $\mathrm{c}_{1}$ & $\lambda^{2}$ \\
\hline 1 & 1 & 0.01 & -0.99 & 0.99 & 1 \\
2 & 1 & 0.019 & -0.98 & 0.171 & 5.7 \\
3 & 1 & 0.029 & -0.97 & 0.101 & 9.6 \\
4 & 1 & 0.039 & -0.96 & 0.071 & 13.382 \\
5 & 1 & 0.049 & -0.951 & 0.055 & 17.071 \\
6 & 1 & 0.058 & -0.941 & 0.045 & 20.68 \\
7 & 1 & 0.067 & -0.936 & 0.038 & 24.215 \\
\hline
\end{tabular}

The output variance of the process described by the model (16) for some $\mathrm{hT}_{\mathrm{S}}$ and $\mathrm{Q}$ as a parameter is plotted in Fig. 4. The asymptotic line represents the maximum value of the output variance which may reach if the process under consideration is uncontrolled.

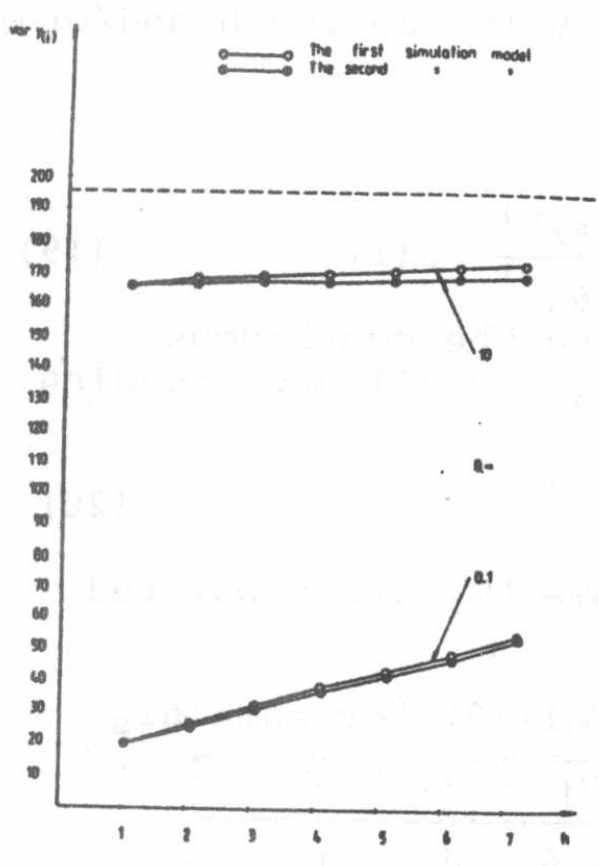

F1g.4. Output variance for some $h \mathrm{~T}_{\mathrm{s}}$ and $Q$ as a parameter for the nodel (16)

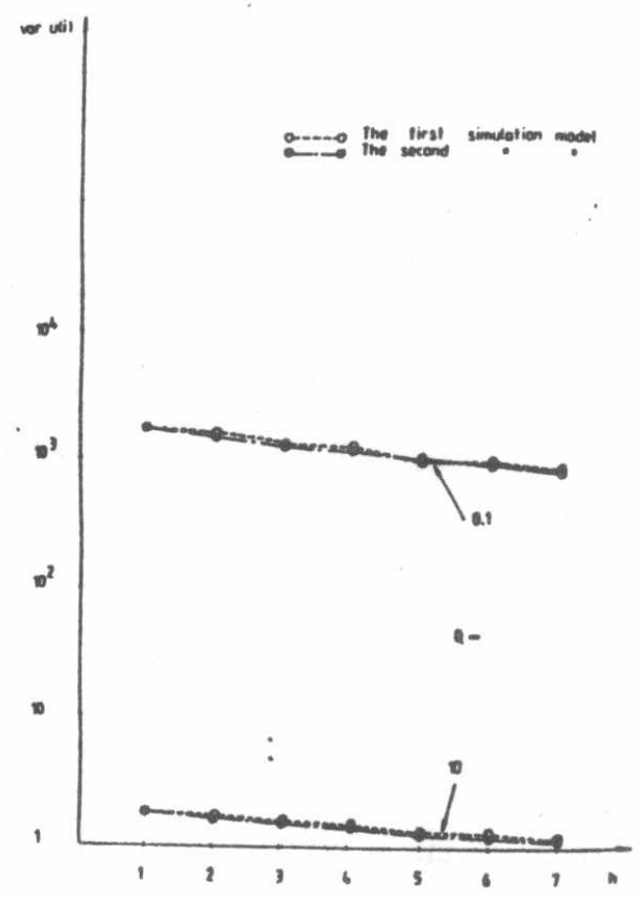

Fig.5. Input variance for some $\mathrm{hT}_{\mathrm{s}}$ and $Q$ as a parameter for the model (16)

From Fig. 4 we see that the output variance increases by increasing $h$ and / for $Q$. In order to have an appreciable evaluation of the control performance under the action of contrllers performing with different sampling intervals, we introduce the fillowing ratio 


$$
(\text { var) } h / \text { Var }
$$

where $(\operatorname{var})_{\mathrm{h}}$ denotes the minimum output variance of the controlled process for a given $h$ and Var denotes the output variance of the process under condideration without control. This ratio is used as a measure of the control performance for different sampling intervals. The ratio (var)h / Var for the process described by the model (16) for some $h$ and $Q$ is tabulated in Table 2 .

Table 2 The ratio (var)h/Var for the process described by the model (16) for some $h$ and $Q$.

\begin{tabular}{|l|l|l|l|}
\hline \multicolumn{4}{|c|}{ (var) $\mathrm{h} /$ Var } \\
\hline $\mathrm{h}$ & $\mathrm{Q}=0$ & $\mathrm{Q}=0.1$ & $\mathrm{Q}=10$ \\
\hline 1 & 0.024 & 0.104 & 0.839 \\
2 & 0.067 & 0.137 & 0.85 \\
3 & 0.104 & 0.167 & 0.864 \\
4 & 0.139 & 0.196 & 0.872 \\
5 & 0.173 & 0.225 & 0.878 \\
6 & 0.206 & 0.252 & 0.882 \\
7 & 0.238 & 0.279 & 0.886 \\
\hline
\end{tabular}

The input variance of the process described by the model (16) for some $h \mathrm{~T}_{\mathrm{S}}$ and $\mathrm{Q}$ as a parameter is plotted in Fig. 5. From this figure we see that the input variance is very large for samll sampling intervals and decreases by increasing $\mathrm{h}$ and/or $\mathrm{Q}$

Example 2

$$
y(i)=z^{-1} \frac{0.4}{1-0.6 z^{-1}} u(i)+\frac{1+0.6 z^{-1}}{1-0.6 z^{-1}} \text { e (i) }
$$

represents the model obtained by sampling the continuous process (17) assuming $\mathrm{T}_{1}=1, \theta=0, \mathrm{~T}_{\mathrm{S}}=0.511$ and assuming that the disturbance is described by

$$
d(i)=\frac{1+0.6 z^{-1}}{1-0.6 z^{-1}} \text { e(i) }
$$

The parameters of the model (19) for some $\mathrm{hT}_{\mathbf{S}}$ are tabulated in Table 3 .

Table 3 Paramerers of the model(19) for some $\mathrm{hT}_{\mathbf{S}}$

\begin{tabular}{|l|l|l|l|l|l|}
\hline $\mathrm{h}$ & $\mathrm{k}$ & \multicolumn{1}{|c|}{$\mathrm{b}_{\mathrm{O}}$} & $\mathrm{a}_{1}$ & $\mathrm{c}_{1}$ & \multicolumn{1}{|c|}{$\lambda^{2}$} \\
\hline 1 & 1 & 0.4 & -0.6 & 0.6 & 1 \\
2 & 1 & 0.64 & -0.3 & 0.143 & 2.517 \\
3 & 1 & 0.784 & -0.216 & 0.072 & 3.98 \\
4 & 1 & 0.87 & -0.129 & 0.041 & 3.156 \\
5 & 1 & 0.922 & -0.077 & 0.024 & 3.216 \\
6 & 1 & 0.953 & -0.046 & 0.014 & 3.238 \\
7 & 1 & 0.972 & -0.028 & 0.008 & 3.24 \\
\hline
\end{tabular}

The output variance of the process described by the model (19) for some $\mathrm{hT}_{\mathrm{S}}$ and $\mathrm{Q}=0$ is plotted in Fig. 6. From this figure 
value for $h \geqq 3$. The ratio $(\operatorname{var})_{h} / \operatorname{Var}$ for the process described by the model (19) for some $h$ and $Q=0$ is tabulated in Table 4. Comparing the values for (var) $/$ Var in Table 2 with the corr esponding values in Table 4 shows the large deterioriation achieved in the control performance by increasing the sampling interval.

Table 4 The ratio (var) $/$ /Var for the process described by the model (19) for some $\mathrm{hT}_{\mathbf{S}}$ and $\mathrm{Q}=0$.

\begin{tabular}{|c|c|}
\hline & $($ var) $\mathrm{h} /$ Var \\
\hline $\mathrm{h}$ & $\mathrm{Q}=0$ \\
\hline 1 & 0.745 \\
2 & 0.949 \\
3 & 0.981 \\
4 & 0.99 \\
5 & 0.994 \\
6 & 0.996 \\
7 & 0.998 \\
\hline
\end{tabular}

The input variance of the process described by the model (19) for some $h$ and $Q=0$ is plotted in Fig. 7. From this figure we see that the input variances are very small compared to the input variances of the model (16) although the weighting factor equals zero.

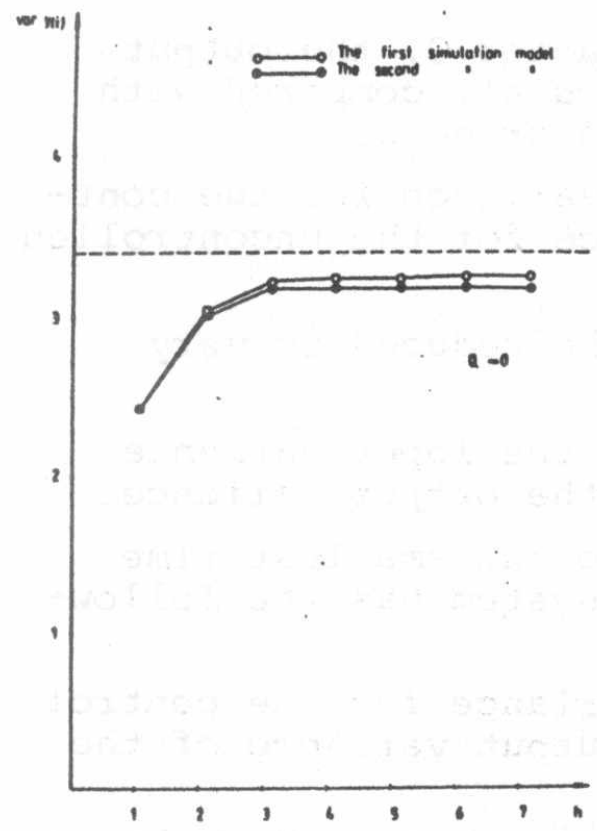

Fig.6. Output variance for some $\mathrm{hT}_{\mathrm{s}}$ and $\mathrm{Q}=0$ for the model (19)

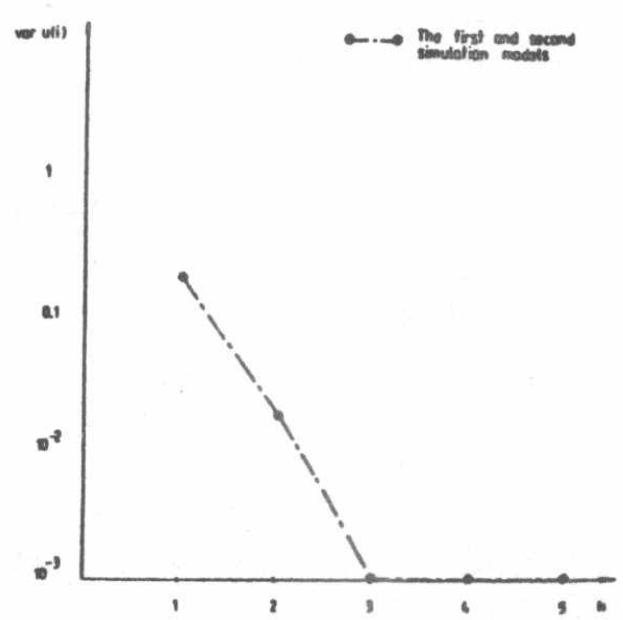

Fig.7. Input variance for some $h T_{s}$ and $Q=0$ for the model (19)

\section{CONCLUSIONS}

The performance of minimum variance control systems was discussed for a set of processes which have been indentified using different sampling intervals and controlled using minimum variance controllexs performing with different sampling intervals. 
The following results are deduced:

1: It is possible to analyze the influence of sampling interval on minimum variance control systems using digital simulation technique.

2. Two simulation models were proposed for the calculations of the input/output variances of the controlled process for sampling intervals that are integer multiples of the basic sampling interval. In the first simulation model, the input/output variances for different $\mathrm{hT}_{\mathrm{S}}$ are calculated using the input/output observations and models for the corresponding $\mathrm{hT}_{\mathbf{S}}$. In the $\mathrm{sec}=$ ond simulation model, the input/output variances for different $\mathrm{hT}_{\mathrm{S}}$ are calculated using the input/output observations and models always for the basic sampling interval.

3. The performance of the minimum variance control system was discussed for the following cases:

A. Sampling intervals small compared to the samllest time constant of the continuous process.

B. Sampling intervals comparable to the smallest time constant of the continuous process.

C. Sampling intervals very large compared to the smallest time constant of the continuous process.

For sampling intervals small compared to the smallest time constant, the minimum variance control system has the following characteristics:

1. For small values of the weighting factor $Q$, the output variance for the control system is very small compared with the output variance for the uncontrolled process.

2. For larger values of $Q$, the output variance for the control system is near to the output variance for the uncontrolled process.

3. The input variance for small $Q$ can be reduced to very small values using large values of $Q$.

4. By increasing the sampling interval the input variance decreases at the expense of increasing the output variance.

B. For sampling intervals comparable to the smallest time constant, the minimum variance control system has the follow ing characteristics:

1. For small values of $Q$ the output variance for the control system is not small compared with the output variance of the uncontrolled process.

2. For large values of $Q$ the output variance for the control system does not increase much more than its value for small values of $Q$. In other words, $Q$ has less influence on the control performance.

3. The input variance is small and can be reduced further using large values of $Q$.

C. For very large sampling intervals, the input variance reaches zero even for $\mathrm{Q}=0$ and the output variance reaches the value of the output variance for the uncontrolled process. 
1. Janiszowski, K., "Synteza cyfrowych ukladow regulacjiwoparciu 0 lacz ocena modelu procesu $i$ doboru regulatora" Wydawnictwa politechiki Warszawskiej, Warsow, Poland (1983). 2. Isermann, R., "Digital Control Systems", Springer, Berlin,
(1981).

3. Åstrom, K. and Wittenmark, B., "Computer Controlled Systems", Prentice-Hall, Inc., Englewood Cliffs (1984).

4. Astrom, K.J., "Introduction to Stochastic Control Theory", Academic Press, New York (1970).

5. Koutb, M.A., "On the Choice of Sampling Interval for Minimum Variance Control Systems",Ph.D. Thesis. Menoufia University, Egypt (1985).

6. Niederlinski, A.,"Wplyw Okresu Probkowania na Parametry Modeli Obiektow Dynamicznych Liniowych Dyskretnych", Automatyka z. 71, Nr Kol. 772. Poland (1983). 